\title{
ANSÄTZE EINER BETRIEBSWIRTSCHAFTLICHEN THEORIE DES VERSICHERUNGSUNTERNEHMENS
}

\author{
von Dieter Farny *
}

\section{Uberblick Uber den Stand der deutschsprachigen Betriebswirtschaftslehre}

Die im deutschsprachigen Raum entwickelte Betriebswirtschaftslehre (BWL) weicht erheblich von den Konzeptionen im angelsächsischen und romanischen Sprachbereich ab. Das zeigt sich unter anderem darin, da $B$ es eine direkte Ubersetzung des Wortes "Betriebswirtschaftslehre" in die englische und französische Sprache nicht gibt ; inhaltlich liegt die deutschsprachige BWL teilweise im Bereich der mikroökonomischen Theorie (economics), teilweise im Bereich der Managementlehre (management science).

Die deutschsprachige BWL hat bereits einen langen Weg hinter sich und sowohl methodisch als auch inhaltlich zahlreiche Veränderungen erfahren. In methodischer Hinsicht sind heute vor allem drei Richtungen zu unterscheiden :

a) Die praktisch-normative BWL gibt Anleitungen zu einem wirtschaftlichen Handeln, welches zur Erreichung von autonom durch das Unternehmen gesetzten Zielen zweckmäßig ist ;

b) Die wertend- normative BWL entwickelt dagegen Zielvorstellungen auf der Grundlage allgemeingültiger Normen und leitet darauf bezogene entsprechende Verhaltensweisen der Unternehmen ab ; sie stellt auch deutlich den Bezug des einzelnen Unternehmens zur Gesamtwirtschaft und zur Gesellschaft her ;

c) Die theoretische BWL entwickelt Erklärungsmodelle für einzelwirtschaftliche Fragen, ohne unmittelbar Handlungsanweisungen abzuleiten.

Die heute am stärksten verbreitete methodische Auffassung ist die von der praktisch-normativen BWL, die wertfrei in dem Sinne ist, daß sie auf empirisch vorhandene Unternehmensziele eingeht, ohne diese im einzelnen zu werten. Insoweit ist diese Ausprägung der BWL vom Wirtschafts- und Gesellschaftssystem unabhängig, in denen sich die Unternehmen befinden.

Inhaltlich bezieht sich die moderne BWL im deutschsprachigen Raum nicht mehr so sehr auf das Unternehmen (oder den Betrieb) als Institution als vielmehr auf das Wirtschaften im Unternehmen. Allerdings sind auch hier die theoretischen, systembildenden Ansätze verschieden, je nachdem, welchem Merkmal des Wirtschaftens die Priorität für die betriebswirtschaftliche Analyse gegeben wird. Infolgedessen können mehrere Ansätze betriebswirtschaftlicher Theorienkonzepte festgestellt werden :

a) Das Wirtschaften im Unternehmen wird als ein Gütereinsatz-, Gütertransformations- und Güterentstehungsproze $B$ gedeutet. Man kann diese Theorie als güterliche $B W L$ bezeichnen; ihr Hauptvertreter ist Gutenberg;

* Professor für Versicherungswirtschaft, Geschäftsführender Direktor des Instituts für Versicherungswissenschaft an der Universität zu Köln. 
b) Das Wirtschaften wird als Summe von Entscheidungen aufgefaßt, nämlich als Entscheidungen über Unternehmensziele und die zu ihrer Erreichung eingesetzten Mittel. Man bezeichnet diese Theorie als entscheidungsorientierte BWL; ihr Hauptvertreter ist Heinen;

c) Das Wirtschaften wird als Aufgabe im Unternehmen aufgefaßt, die in Teilaufgaben zerlegt werden kann. Diese wiederum werden durch wirtschaftliche Prozesse im institutionellen Rahmen des Unternehmens erfüllt. Diese Betrachtungsweise führt zu einer funktionalen $B W L$, die als das Integral von Partialanalysen der Teilaufgaben zu verstehen ist;

d) Das Wirtschaften im Unternehmen wird als System aufgefaßt und mit Hilfe der allgemeinen Systemtheorie und neuerdings auch der Kybernetik untersucht. Diese noch sehr formale Betrachtungsweise wird als systemtheoretische $B W L$ bezeichnet; ihr Hauptvertreter ist der Schweizer Ulrich.

Eine Dominanz einer dieser betriebswirtschaftlichen Konzeptionen ist derzeit nicht eindeutig feststellbar; im deutschsprachigen Lehr- und Forschungsbetrieb der BWL werden alle genannten Richtungen vertreten.

\section{Deduktionen fur elne betriebswirtschaftliche Theorle des Versicherungsunternehmens}

Die BWL hat ihre Theorien vorwiegend für den sachgütererzeugenden Industriebetrieb entwickelt. Das entspricht der historischen Bedeutung dieser Unternehmensgruppe in der bisherigen Geschichte der BWL. Erst in jüngerer Zeit, seitdem die Bedeutung des tertiären Sektors (Dienstleistungsbereich) zunehmend wächst, werden auch Theorien über Dienstleistungsunternehmen, darunter auch die Versicherungsunternehmen, erarbeitet.

Dabei ist es nicht erforderlich, für einzelne Typen von Dienstleistungsunternehmen von Grund auf neue betriebswirtschaftliche Theorien zu erarbeiten, sondern in vielen Fällen führt bereits eine Deduktion allgemein-betriebswirtschaftlicher Hypothesen zu brauchbaren Ergebnissen. Mit dieser Methode lassen sich auch Aussagen über eine betriebswirtschaftliche Theorie des Versicherungsunternehmens gewinnen. Voraussetzung für solche Schlüsse ist, $\mathrm{da} ß$ in Versicherungsunternehmen gewirtschaftet wird, also planmäßig Prozesse vollzogen werden, die zur Entstehung von Guitern für die Befriedigung menschlicher Bedürfnisse führen. Es bedarf wohl keiner eingehenden Beweise dafür, daß diese Voraussetzung vollkommen gegeben ist ; denn Versicherungsschutz ist ein reales, immaterielles Wirtschaftsgut, das dem Verwender einen Nutzen stiftet, knapp ist und einen Wert besitzt. Er wird im Versicherungsunternehmen durch Einsatz und Kombination anderer Wirtschaftsgüter produziert (güterliche BWL). Dieser Produktionsproze $B$ ist kein Automatismus, sondern wird durch Entscheidungen über Unternehmensziele und über Mittel ausgelöst, gesteuert und gegebenenfalls beendet (entscheidungsorientierte BWL). Betrachtet man ihn als die Gesamtaufgabe im Versicherungsunternehmen, so lassen sich Teilfunktionen erkennen, die durch Prozesse im institutionellen Rahmen des Unternehmens erfüllt werden (funkionale BWL). Formal kann man schließlich das Versicherungsunternehmen auch als ein offenes System verstehen, dessen Elemente miteinander verknüpft sind und eine gewisse Ordnung (Struktur) aufweisen (systemtheoretische BWL). 


\section{Güterllche Theorle des Verslcherungsunternehmens}

\subsection{Ubersicht}

Eine auf die Güterprozesse im Versicherungsunternehmen ausgerichtete betriebswirtschaftliche Theorie hat den Einsatz der Produktionsfaktoren, die Kombination der Produktionsfaktoren und die Entstehung der Versicherungsprodukte zum Gegenstand. Sie untersucht Güterströme und Güterbestände im Versicherungsunternehmen. Der Kern der Theorie ist die Input-Output-Relation, in der die Produktivitätsbeziehung zum Ausdruck kommt. Soweit die mengenmäßige Input-Output-Beziehung erfaßt werden soll, spricht man von der Produktionstheorie, wogegen die Relation von wertmäßigen Input (Kosten) und Güteroutput als Kostentheorie bezeichnet wird.

\subsection{Input (Produktionsfaktoren)}

Die im Versicherungsunternehmen eingesetzten Produktionsfaktoren werden auf den Beschaffungsmärkten beschafft (originäre Produktionsfaktoren) oder im Unternehmen selbst hergestellt (derivative Produktionsfaktoren). Nach Güterarten klassifiziert, handelt es sich vor allem um folgende Einsatzgüter :

a) Physische und intellektuelle Arbeitsleistungen (besonders Mitarbeiter);

b) Dienstleistungen dritter Betriebe (besonders Vermittlungsdienstleistungen);

c) sachliche Betriebsmittel (besonders Gebäude, Einrichtungen, Datenverarbeitungsanlagen);

d) Hilfs- und Betriebsstoffe ;

e) Geld für Versicherungsleistungen;

f) Rückversicherung ;

g) Kapitalnutzungen (Vorrätigkeit an Nominal- und Realgütern einschließlich Sicherheismitteln).

In diesem Input des Versicherungsunternehmens zeigen sich vor allem zwei Besonderheiten : erstens bedarf es zur Produktion von Versicherungsschutz keiner Rohstoffe, da das Produkt immaterieller Natur ist, und zweitens ist das Nominalgut Geld in seiner Originalform als Produktionsfaktor verwendbar. Das letztere ist die Folge des in aller Welt vorherrschenden Prinzips, daß Versicherungsleistungen in Geldform gewährt werden; würde der Versicherer Naturalersatz leisten, dann müßte er entsprechende Realgüter beschaffen und nach Einfügung in den Produktionsproze $B$ an den Versicherungsnehmer weiterleiten.

\subsection{Transformation (Produktionsfaktorkombination)}

Die Produktionsfaktorkombination im Versicherungsunternehmen ist in beträchtlichem Ausmaß gestaltungsfähig. Nur ein Teil der Produktionsfaktoreinsätze ist limitational an den Output oder an andere Produktionsfaktoreneinsätze gebunden, ein anderer Teil ist dagegen in Grenzen oder sogar völlig frei wechselseitig substituierbar. Das letztere gilt zum Beispiel für das Verhältnis von Geld für Versicherungsleistungen für eigene Rechnung (Selbstbehalt) und Rückversicherung. Aus diesen substitutionalen Verhältnissen entsteht das Optimierungsproblem bei der Bestimmung der Produktionsfunktionen, die die Faktoreinsätze in Abhängigkeit vom gewünschten Output angibt. Es handelt sich dabei um Fragen der klassischen Versicherungstheorie, insbesondere 
der Gestaltung des Risikoausgleichs im Kollektiv und in der Zeit, die nicht zuletzt in quantitativen Modellen gelöst werden können; in diesem Zusammenhang gibt es bereits einige Anwendungen des Operations Research.

Die Produktionsfaktorkombination führt nicht in einem Zug vom Einsatz der Produktionsfaktoren zum fertigen Versicherungsprodukt. Vielmehr lassen sich die Produktionsprozesse als Vielzahl von einzelnen Transformationsvorgängen auffassen, deren Ergebnisse in Form innerbetrieblicher Leistungsergebnisse letztlich zum Versicherungsschutz führen. Dabei können Prozesse zur Herstellung der betrieblichen Leistungsbereitschaft von solchen zur Herstellung des absatzreifen oder abgesetzten Versicherungsprodukts unterschieden werden.

Ein weiterer wichtiger betriebswirtschaftlicher Teilaspekt der Produktionsfaktorkombination ist die Analyse der Produktionskosten in Abhängigkeit von ihren Bestimmungsgrößen. Nach den bisher bekannten Hypothesen werden die Kosten im Versicherungsunternehmen vor allem von der Betriebsgröße und dem Beschäftigungsgrad, von der Bestandszusammensetzung, von den Faktorqualitäten und den Faktorpreisen bestimmt. Die Forschungsmethoden für diese kostentheoretischen Fragestellungen können unmittelbar aus der allgemeinen BWL übernommen werden.

\subsection{Output (Versicherungsprodukte)}

Der Output im Versicherungsunternehmen besteht aus den Versicherungsprodukten der verschiedensten Art. Gelegentlich werden gleichzeitig auch weitere Dienstleistungen produziert. Insbesondere liegt in der Realität eine verbundene Produktion von Versicherungsschutz und Kapitalnutzungen vor; praktisch alle Versicherer produzieren Kapital- unr Mietnutzungen, die durch das Stichwort der Kapitalanlagen umschrieben werden.

Das Produkt Versicherungsschutz weist eine Reihe von typischen Merkmalen aller Dienstleistungen, daneben jedoch auch weitere besondere Kennzeichen auf. Er ist als Endprodukt nicht lagerfähig. Deshalb kann Versicherungsschutz erst endgültig und bis zur vollständigen Reife produziert werden, nachdem er verkauft worden ist. Wegen seiner immateriellen Natur bedarf es der Festlegung des Produkts durch Daten und der Konkretisierung durch ein Trägermedium (Papier). Die Datenbezogenheit des Versicherungsprodukts hat dazu geführt, daß häufig das mit dem Produktionsproze $B$ verbundene Datensystem (Datenbeschaffung, Dateninput, Datentransformation, Datenoutput) besondere betriebswirtschaftliche Untersuchungen erfährt. Eine weitere Besonderheit der Versicherungsprodukte ist ihre Zeitraumbezogenheit; infolgedessen sind auch zahlreiche Produktionsfaktoreinsätze und -kombinationsprozesse permanent vorzunehmen.

\subsection{Zusammenfassung.}

Insgesamt liegen für eine gutterliche Theorie des Wirtschaftens im Versicherungsunternehmen bereits heute viele Ansätze vor, insbesondere soweit es den Risikoausgleichsprozeß betrifft. Dagegen stecken die produktionstheoretischen Ansätze für die übrigen Input-Output-Beziehungen noch in den Kinderschuhen. Dies entpricht dem allgemein zu beobachtenden Rückstand in der betriebswirtschaftlichen Erforschung von Dienstleistungsunternehmen. 
Die Vielfalt des güterlichen Konzepts einer Theorie des Versicherungsunternehmens läßt sich anhand einer Übersicht über die Real- und Nominalgüterströme in einem Versicherungsunternehmen bzw. zwischen dem Unternehmen und seiner Umwelt erkennen (Abbildung 1). Die auffälligste Besonderheit liegt darin, da $\beta$ das Nominalgut Geld, soweit es für Versicherungsleistungen eingesetzt wird, nicht auf den Beschaffungsmärkten in materielle Einsatzgüter umgewandelt werden muß, wie dies in fast allen anderen Wirtschaftszweigen erforderlich ist, wenn ein produktiver Effekt erzielt werden soll.

\section{Entscheidungsorientierte Theorle des Versicherungsunternehmens}

\subsection{Ubersicht}

Das Wirtschaften im Versicherungsunternehmen ist kein Automatismus, sondern wird durch Entscheidungen von Menschen ausgelöst und gesteuert. Wirtschaften bedeutet also die Entscheidung über den Einsatz knapper Mittel zur Erreichung von Zielen. Die betriebswirtschaftliche Theorie führt deshalb zur Erklärung und Gestaltung von Ziel-Mittel-Relationen.

Wie in jedem Unternehmen betrifft die alle weiteren wirtschaftlichen Aktivitäten bestimmende Entscheidung die Ziele, welche mit dem Wirtschaften verfolgt werden. Diese Ziele können nach heutiger betriebswirtschaftlicher Auffassung weder als Normen vorgegeben werden, noch unterliegen sie einer wertenden Kritik des Betriebswirts. Dieser kann lediglich den empirischen Zieltatbestand feststellen. Mit der Zielentscheidung sind praktisch alle weiteren wirtschaftlichen Handlungen im Versicherungsunternehmen festgelegt; es handelt sich nur noch um die Auswahl der zielentsprechend optimalen Strategien, unter denen die Programmentscheidungen und die Verfahrensentscheidungen die wichtigsten bilden.

\subsection{Zielentscheidungen}

Die Erforschung empirisch vorhandener Ziele von Versicherungsunternehmen ist bisher über die Aufstellung von Hypothesen nicht weit hinausgekommen. Unternehmensziele sind angestrebte Zustände. Entsprechend der Beobachtung der Realität kann angenommen werden, da $B$ Versicherungsunternehmen unter anderem nach der Deckung von Versicherungsbedarf, nach Erzielung von Gewinn, nach Umsatz oder Marktanteil sowie nach zahlreichen nichtmonetären Größen und Zuständen (z.B. Prestige, Macht, Unabhängigkeit) streben, jeweils unter der Bedingung einer dauerhaft gesicherten Existenz. Die Schwierigkeiten der Zielentscheidung bestehen in zahlreichen komplementären und konkurrierenden Beziehungen unter den Einzelzielen. In den meisten Ländern ist die Autonomie der Zielentscheidung durch rechtliche, besonders aufsichtsrechtliche, Daten begrenzt. Dies gilt vor allem für Gewinnziele. Die Analyse der an der Zielentscheidung beteiligten Entscheidungsträger (Eigentümer, Unternehmensleitung, Versicherungsnehmer, Belegschaft, Aufsichtsbehörde, Staat) führt von betriebswirtschaftlichen zu sozialwissenschaftlichen Fragestellungen, die im Zusammenhang mit der Zielanalyse verbreitet Eingang in die BWL gefunden haben.

\subsection{Programmentscheidungen}

Nach Festlegung der Unternehmensziele folgt logisch die Entscheidung über das Produktionsprogramm; sie soll das für die Zielerfüllung günstigste Produktionspro- 
gramm bestimmen und umfaßt sowohl die materielle und formale Gestaltung der einzelnen Versicherungsprodukte als auch ihre Zusammenfassung in einem Sortiment. Die Prüfung der Zielwirkungen der Programmentscheidung betrifft vor allem Umsatzund Gewinnerwartungen sowie das $\mathrm{Maß}$ des versicherungstechnischen Risikos; im letzten Fall interessiert vor allem die Gesamtschadenverteilung des vorgesehenen Versicherungsbestandes, von der das Unternehmensrisiko bzw. die Existenzsicherheit maßgeblich beeinflußt wird.

Bei der Programmentscheidung sind üblicherweise zahlreiche aufsichtsrechtliche Vorschriften $\mathrm{zu}$ beachten, so etwa bei der Gestaltung von Versicherungsbedingungen sowie bei den weitverbreiteten Spartenkombinationsverboten. In den meisten Ländern ist beispielsweise der Betrieb der Lebensversicherung nur in einer eigenen Rechtseinheit möglich, was zur verbreiteten Bildung von Versicherungskonzernen geführt hat, wenn ein generelles Produktionsprogramm angestrebt wird. Die Programmentscheidung legt auch den Grad der Spezialisierung bzw. Generalisierung der Geschäftstätigkeit des Versicherungsunternehmens fest; Spezialisierung kann auf Versicherungszweige, auf Kundengruppen oder regionale Geschäftsgebiete bezogen sein.

Neuerdings werden Programmentscheidungen unter anderem von der Frage geprägt, ob das Versicherungsunternehmen auch versicherungsfremde Produkte in sein Sortiment aufnehmen soll; diskutiert werden zum Beispiel Finanzdienstleistungen, Schadenverhütungsdienstleistungen und ähnliches. Der betriebswirtschaftliche Gehalt einer solchen Diversifikation rührt vor allem aus der Verbundenheit der Nachfrage nach Versicherungsprodukten und anderen Dienstleistungen sowie aus der Möglichkeit einer verbundenen Produktion; denn viele im Versicherungsunternehmen eingesetzte Produktionsfaktoren können auch zur Produktion weiterer Dienstleistungen verwendet werden.

\subsection{Verfahrensentscheidungen}

Nach Festlegung von Unternehmenszielen und Produktionsprogramm folgt die Entscheidung über das Produktionsverfahren. Insbesondere sollen die für die Zielerreichung optimalen Verfahren gefunden werden, wobei die Minimierung der Produktionskosten im Vordergrund der betriebswirtschaftlichen Fragestellung steht. "Verfahren" ist in diesem Zusammenhang ganz allgemein $\mathrm{zu}$ verstehen und betrifft sowohl die Gestaltung des Risikoausgleichsprozesses als auch die Durchführung einzelner betriebswirtschaftlicher Leistungsprozesse im Bereich der Vertragsbearbeitung, der Schadenbearbeitung, des Absatzes, der Finanzen und anderer Teilaufgaben. Besonderes Interesse hat in den letzten Jahren den Entscheidungen über Datenverarbeitungsverfahren gegolten; hier zeigt sich abermals, daß im Versicherungsunternehmen das Teilsystem der versicherungsvertragsbezogenen und der versicherungsfallbezogenen Daten sehr gut gesonderter betriebswirtschaftlicher Analyse zugänglich ist.

\subsection{Zusammenfassung}

Der entscheidungsorientierte Ansatz für eine betriebswirtschaftliche Theorie des Versicherungsunternehmens umfaßt also zwei Gesichtspunkte : erstens die Erforschung empirischer Zielvorstellungen im Versicherungsunternehmen und zweitens die Untersuchung der kausalen und finalen Beziehungen zwischen den Unternehmenszielen einer- 
seits und den programm- und verfahrensstrategischen Alternativen andererseits. Letztlich gilt dieser Ansatz einer betriebswirtschaftlichen Theorie der Suche nach dem teleologisch optimalen Mitteleinsatz im Versicherungsunternehmen.

\section{Funktionale Theorle des Versicherungsunternehmens}

\section{1. Übersicht}

Das Wirtschaften im Versicherungsunternehmen ist kein einfach strukturierter Vorgang, sondern es setzt sich aus einer Vielzahl von Elementen zusammen. Betriebswirtschaftliche Totalanalysen sind deshalb im Regelfall entweder nur formal oder aber zu oberflächlich, als daß unmittelbare Anweisungen zum zweckmäßigen wirtschaftlichen Verhalten gewonnen werden könnten. Diese Einsicht hat dazu geführt, daß in der BWL die Gesamtaufgabe des Wirtschaftens in betriebswirtschaftliche Teilaufgaben gegliedert wurde, um diese für Partialanalysen mit besseren Erkenntnismöglichkeiten zugänglich zu machen. Jede der betriebswirtschaftlichen Teilfunktionen kann wiederum güterlich, also als Input-Transformations-Output-System, begriffen oder unter dem Aspekt der Entscheidungen untersucht werden. Die Interpretation des Wirtschaftens als der Summe von Teilaufgaben eröffnet auch die Fragestellung nach der Organisation des Versicherungsunternehmens; denn Organisation wird üblicherweise als das System von Regelungen für die Erfüllung von Aufgaben verstanden.

Systeme betriebswirtschaftlicher Teilfunktionen sind in großer Zahl entwickelt worden. Für die hier beabsichtigte Ưbersicht genügt die Einteilung in nach außen gerichtete Funktionen (Beschaffung, Absatz) und in interne Funktionen (Leistungserstellung, Verwaltung); eine Sonderstellung nimmt im Funktionensystem die Finanzierungsfunktion ein, die sich auf die Gesamtheit aller Nominalgütervorgänge erstreckt.

\subsection{Beschaffung}

Die nach außen, also in die Umwelt des Versicherungsunternehmens gerichteten Funktionen sind Beschaffung und Absatz. Die marktmäßige Beschaffung betrifft alle Produktionsfaktoren, erstreckt sich somit unter Umständen auf Märkte sehr verschiedener Art. In der Praxis sind vor allem Personal-, Sachmittel- und Rückversicherungsbeschaffung von besonderer Bedeutung.

\subsection{Absatz}

Eine der bisher am besten untersuchten Teilfunktionen ist der Absatz des Versicherungsunternehmens. Absatz bedeutet die Abgabe der Versicherungsprodukte am Markt gegen Zahlung eines Preises, der Prämie. Dieser Tatbestand eröffnet eine Fülle betriebswirtschaftlicher Fragestellungen. Beispielsweise ist die Absatzvorbereitung (Informationsgewinnung, Absatzplanung) von der Absatzdurchführung zu unterscheiden. Die Absatzdurchführung hat die Aufgabe, die Distanzen räumlicher, quantitativer, qualitativer, zeitlicher und informationsmäßiger Art zwischen den Kunden und dem Versicherer abzubauen. Der Abschluß einer Versicherung kommt nach einem einfachen ökonomischen Erklärungsmodell zustande, wenn beim Versicherungsnehmer ihr Nutzen höher eingeschätzt wird als die Prämienausgabe und wenn gleichzeitig beim Versicherer der Abschluß der Versicherung die Erreichung der Unternehmensziele fördert. Allein 
die wirtschaftliche Analyse dieses Verhältnisses und die Untersuchung der Einflußmöglichkeiten in diesem Bereich bedeuten hochrangige betriebswirtschaftliche Probleme.

Ein weiteres betriebswirtschaftliches Kernproblem ist die instrumentale Seite des Absatzes, die Absatzpolitik. Gefragt wird nach den Instrumenten zur Beeinflussung der betriebsindividuellen Absatzsituation und die Kombination der Instrumente zum sogenannten Marketing-Mix. Die Parameter der Absatzpolitik in der Versicherungswirtschaft sind recht eigenartig ausgeprägt. Die Absatzprogrammpolitik fragt nach Produkten und Sortimenten mit hohem akquisitorischen Potential ; Teil der Programmpolitik ist auch die Gestaltung des Services gegenüber den Kunden, weil Qualität und Quantität des Service sowohl Umsatz als auch Kosten beeinflussen. Die Preispolitik ist in ihrer Wirkung begrenzt; einmal bezieht sie sich vorwiegend nur auf das Neugeschäft, zum anderen ist die Autonomie der Versicherungsunternehmen auf diesem Gebiet vielfach durch aufsichtsrechtliche oder andere Vorschriften begrenzt. Wichtige betriebswirtschaftliche Einzelfragen in diesem Bereich sind die Feststellung von Preisuntergrenzen für einzelne Geschäfte und die Gestaltung der einzelnen preisbestimmenden Parameter (Tarifprämien, Verhandlungsprämien, Prämienrückerstattung, Inkassoverfahren, Prämienzahlungsweise, Erfahrungstarifierung). Neuerdings sind Prämienanpassungsklauseln zum beherrschenden Thema der Preispolitik geworden. Die Werbungspolitik legt den Informationsstrom fest, der vom Versicherer an die Kunden zur Beeinflussung ihres Nachfrageverhaltens fließt; dabei sind viele einzel- und sozialpsychologische Fragen zu berücksichtigen.

Das in der Realität an Bedeutung wichtigste absatzpolitische Instrument ist die Auswahl des Absatzverfahrens. Darunter versteht man die Auswahl der Absatzorgane (Versicherungsvermittler) und die Festlegung der Regelungen für den Einsatz der Absatzorgane. Die Absatzverfahrensalternativen sind jeweils vor allem im Hinblick auf Umsatz- und Kostenziele zu würdigen. Für die Entscheidungen über Absatzverfahren liegen viele betriebswirtschaftliche Arbeiten und auch große praktische Erfahrungen vor.

\subsection{Leistungserstellung}

Die wichtigste interne Kernfunktion im Versicherungsunternehmen kann als Leistungserstellung gekennzeichnet werden. Dazu gehören insbesondere die auf einzelne Versicherungsverträge, Versicherungsfälle oder auf den Gesamtversicherungsbestand bezogenen Aufgaben, also die Vertragsbearbeitung (Erst- und Folgebearbeitung) und die Schadenberarbeitung, sowie die jeweils erforderlichen Vorbereitungen. Gerade diese Aufgaben lassen sich primär als Datenprozesse begreifen; sie sind bevorzugter Anwendungsbereich der operativen automatisierten Datenverarbeitung. Die Gestaltungsfragen beziehen sich vor allem auf die Entscheidungen für automatisierte oder "manuelle" Arbeitsprozesse, den Grad der Spezialisierung bei der Aufgabenerfüllung, die Probleme von Zentralisierung oder Dezentralisierung der einzelnen Arbeitsabläufe, ganz allgemein die Regelungen von Aufbau des Versicherungsunternehmens und den darin sich vollziehenden Abläufen.

Es ist erneut darauf hinzuweisen, daß die Leistungserstellung, die etwa der Fertigung in Industriebetrieben entspricht, im Versicherungsunternehmen erst dann abschließend durchgefuhrt werden kann, wenn die einzelnen Versicherungsprodukte abgesetzt wurden ; eine Lagerwirtschaft für Versicherungsprodukte gibt es deshalb nicht. 


\subsection{Verwaltung}

Die Verwaltungsfunktion umfaßt die nicht unmittelbar auf den Versicherungsbestand, die einzelnen Versicherungsverträge und Versicherungsfälle bezogenen Aufgaben. Sie ist deshalb nur gering versicherungsspezifisch ausgeprägt. Im Vordergrund steht die Personalverwaltung, die Verwaltung der Sachmittel, die Datenverwaltung und das Rechnungswesen.

\subsection{Finanzierung.}

Die Güterströme und -bestände in einem Versicherungsunternehmen betreffen einmal die Realgüter, zum anderen die Nominalgüter (Geld und Forderungen). In der BWL wird ubblicherweise die Gesamtheit der auf Nominalgüter bezogenen Aufgaben als Finanzierungsfunktion zusammengefaßt. Die Finanzierung als Teilaufgabe im Versicherungsunternehmen wird also nach einem anderen Kriterium abgegrenzt als die übrigen erwähnten Teilfunktionen. Sie überspannt gleichsam die anderen Funktionen, soweit diese mit Nominalgüterströmen oder -beständen verbunden sind.

Finanzierung bedeutet insbesondere die Ermittlung des Kapitalbedarfs, der durch Auszahlungen zur Beschaffung von Realgütern oder durch Widmung von Geld zur Vorrätigkeit hervorgerufen wird, und die Deckung des Kapitalbedarfs durch einen bedarfsgerechten Kapitalfonds, der durch verschiedene Finanzierungsformen gespeist werden kann. Dabei unterliegen Versicherungsunternehmen häufig einschneidenden Rechtsvorschriften; auf die Solvabilitätsregelungen im Zusammenhang mit der Niederlassungsfreiheit in der Europäischen Wirtschaftsgemeinschaft sei besonders hingewiesen. Die Analyse der Finanzierungsfunktion in Versicherungsunternehmen zeigt zahlreiche Besonderheiten, zum Beispiel die Verwendung von Geld als Produktionsfaktor, die große Bedeutung immaterieller Investitionen (z.B. in der Außenorganisation, in den Abschlußkosten für den Versicherungsbestand) sowie die übliche Prämienvorauszahlung durch die Kunden. In der Lebensversicherung kommt als wichtiger finanzwirtschaftlicher Tatbestand die Integration von Spar- und Entsparprozessen in das Versicherungsgeschäft hinzu. Prämienvorauszahlung und Sparprozesse führen zu einem tendenziell hohen Bestand an Geld, das in Form von Kapitalanlagen bis zur Auszahlung der Versicherungsleistungen rentabel angelegt wird; hier liegt die Begründung für die bereits erwähnte verbundene Produktion von Versicherungsschutz und Kapitalanlagen, die zu schwierigen Problemen der Zurechnungen von Erträgen und Aufwendungen der beiden Geschäftsbereiche führt.

\section{Systemtheorle des Versicherungsunternehmens}

Ein systemtheoretischer Ansatz für Versicherungsunternehmen liegt bisher erst in Ansätzen vor. Persönlich habe ich Zweifel, ob er außer dem Vorteil formaler Bündigkeit weitere materielle Erkenntniszuwächse bietet. Es ist zu vermuten, daß ein systemtheoretisches Modell des Versicherungsunternehmens vor allem didaktische Vorzuige aufweist, weil es eine hohe Geschlossenheit und die Vollständigkeit des theoretischen Konzepts ermöglicht. 


\section{Integration der betriebswirtschaftlichen Ansătze für eine Theorle des Versicherungsunternehmens}

Die zuvor erwähnten und grob skizzierten Ansätze für eine betriebswirtschaftliche Theorie des Versicherungsunternehmens stehen keineswegs isoliert nebeneinander. Sie bieten lediglich verschiedene Blickrichtungen auf dasselbe Erkenntnisobjekt, nämlich das Wirtschaften im Versicherungsunternehmen, und setzen verschiedene Schwerpunkte für die Theorienbildung.

Güterlicher, entscheidungstheoretischer und funktionaler Ansatz für das Wirtschaften im Versicherungsunternehmen lassen sich wie folgt zusammenfassen (Abbildung 2). Die Untersuchung der Entscheidungen betrifft die Unternehmensziele des Versicherungsunternehmens, die Produktionsprogramme und die Produktionsverfahren. Diese drei Entscheidungsbereiche sind durch zahlreiche, zum Teil recht komplizierte kausale oder finale Beziehungen miteinander verknüpft. Das Produktionsprogramm umschreibt gleichzeitig den planmäßigen Güteroutput, während die Produktionsverfahren mit der Gestaltung des Gütertransformationsprozesses übereinstimmt. Auf der anderen Seite ist die programmgemäße Versicherungsproduktion die Gesamtaufgabe des Unternehmens, die in Teilaufgaben gegliedert wird, welche wiederum durch Prozesse in betrieblichen Institutionen erfüllt werden.

Das Untersuchungsobjekt ist also in allen drei theoretischen Konzepten gleich. Lediglich die Betrachtungsweise ist verschieden, so daß zwar verschiedene theoretische Aussagen gewonnen werden, die jedoch ohne Schwierigkeiten zu einem Gesamtbild, das heißt $\mathrm{zu}$ einer einheitlichen betriebswirtschaftlichen Theorie des Versicherungsunternehmens, integriert werden können. 


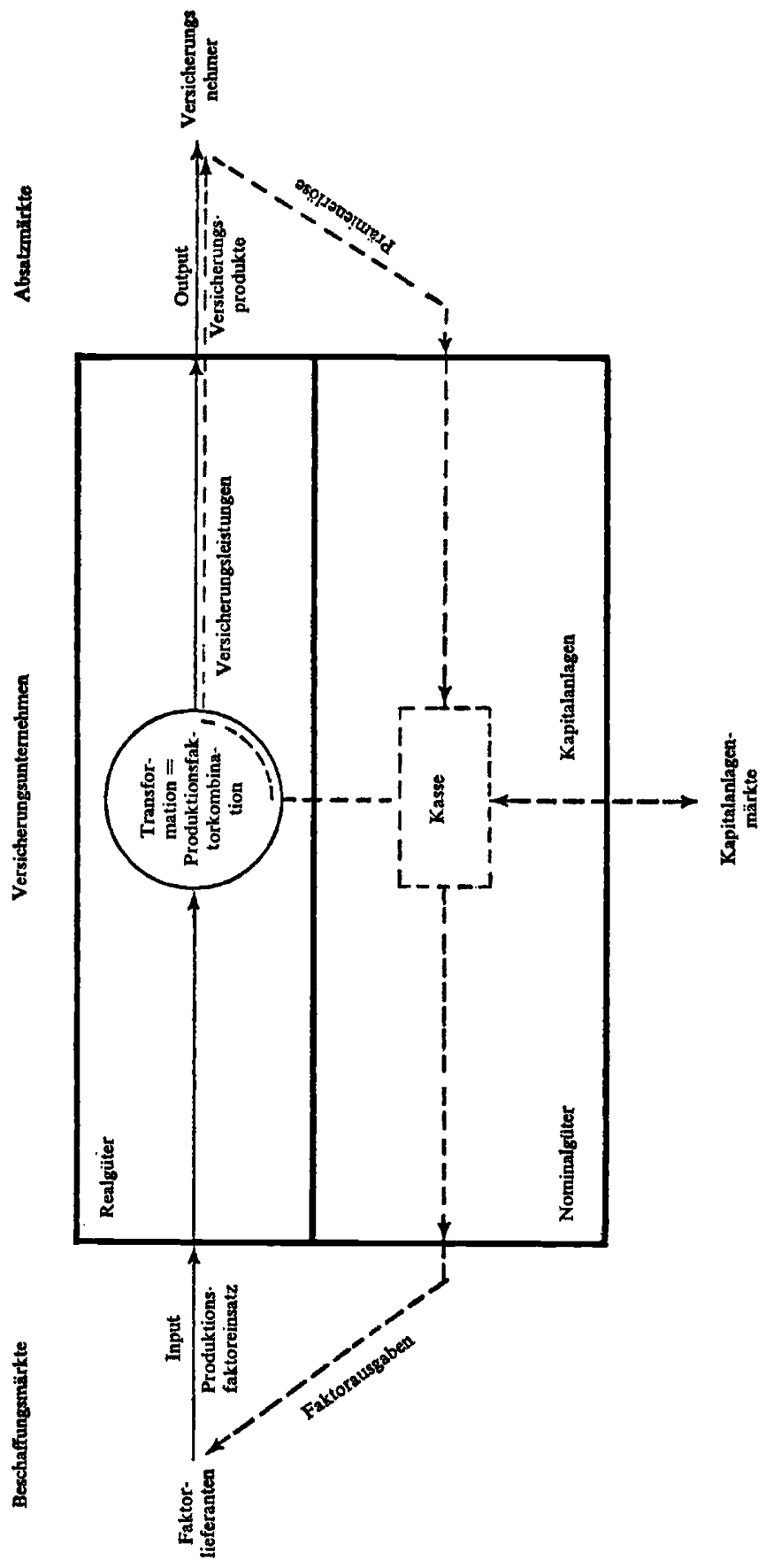

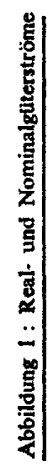




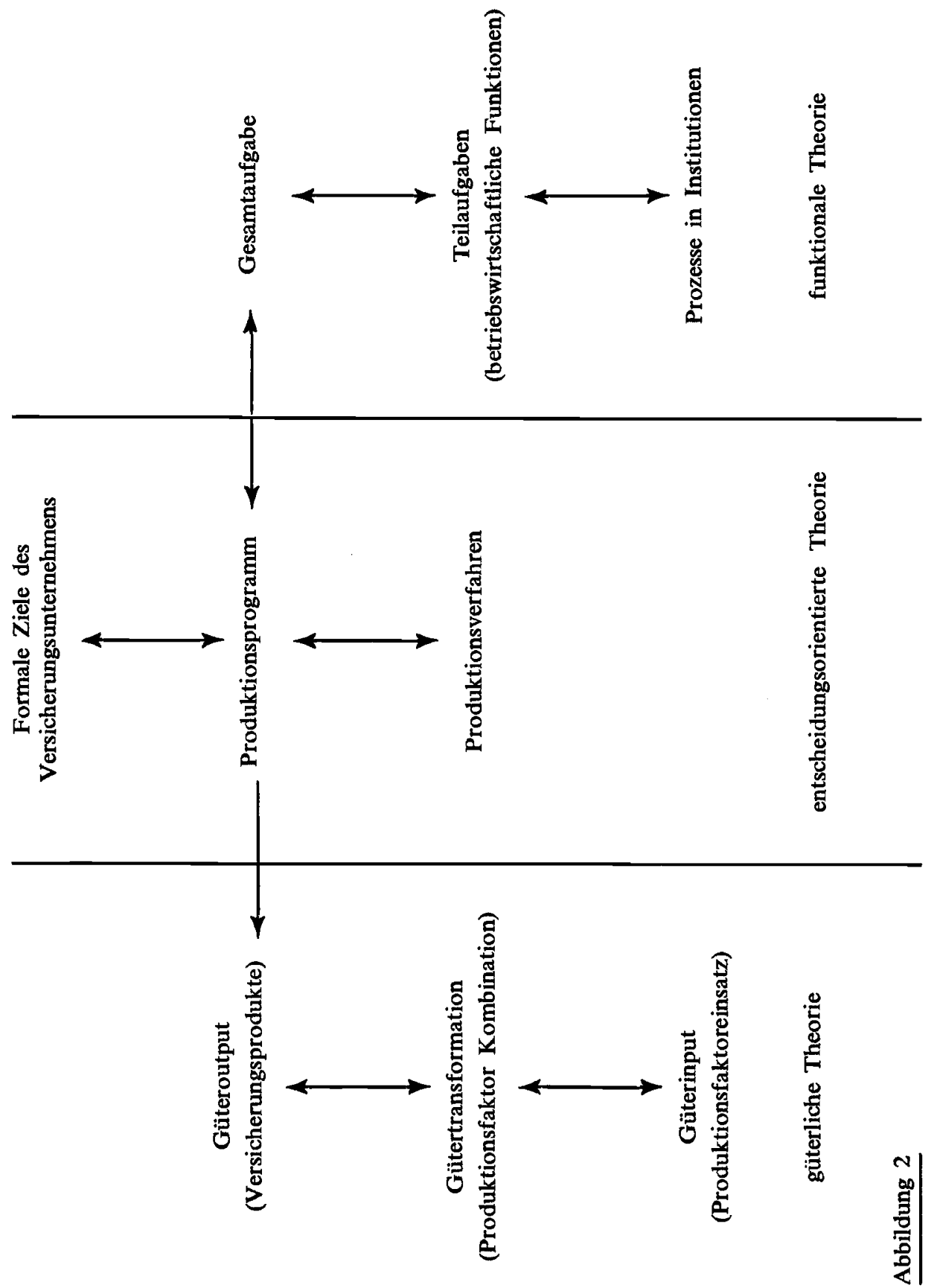




\section{LITERATURHINWEISE}

1. BORCH, K.: “The economic theory of insurance ", ASTIN Bulletin, Vol. IV, Part III, July 1967, 252.

2. BORCH, K. : "Dynamic decision problems in an insurance company", ASTIN Bulletin, Vol. V, Part I, May 1968, 118.

3. CASSANDRO, P.E., Le gestioni assicuratrici, Utet, Torino, 1957.

4. EISEN, R., "Zur Produktionsfunktion der Versicherung”, Zeitschrift für die gesamte Versicherungswissenschaft, 1971, 407.

5. FARNY, D., Produktions- und Kostentheorie der Versicherung, Karlsruhe, 1965.

6. FARNY, D., "Grundfragen einer theoretischen Versicherungsbetriebslehre", in Festschrift Paul Braeß, Karlsruhe, 1969, 27.

7. FOURASTIÉ, J., Les assurances au point de vue économique et social, Payot, Paris, 1946.

8. GURTLER, M., Einführung in die Betriebswirtschaftslehre der Versicherung, Stuttgart, 1964.

9. HAX, K., "Auf dem Wege zu einer Versicherungsbetriebslehre", in Festschrift H.L. Müller-Lutz, Karlsruhe, 1972, 135.

10. KALUZA, Spieltheoretische Modelle und ihre Anwendungsmöglichkeiten im Versicherungswesen, Berlin, 1972.

11. ROHRBECK, Die Versicherungsunternehmung, ihr wirtschaftlicher Sinn und ihr organisatorischer Aufbau, Leipzig, Berlin, 1936.

12. SAMUELSON / ROCKWELL, An application of systems simulations to insurance operations, Columbus, 1967.

13. TRÖNDLE, Grundgedanken zu einem systemtheoretischen Strukturkonzept der Versicherungsunternehmung, Dissertation, Köln, 1974. 